\title{
Many-pole model of inelastic losses in x-ray absorption spectra
}

\author{
J. J. Kas, ${ }^{1}$ A. P. Sorini, ${ }^{1}$ M. P. Prange,${ }^{1}$ L. W. Cambell, ${ }^{2}$ J. A. Soininen, ${ }^{3}$ and J. J. Rehr ${ }^{1}$ \\ ${ }^{1}$ Dept. of Physics, Univ. of Washington Seattle, WA 98195 \\ ${ }^{2}$ Pacific Northwest National Lab. Richland, WA 99352 \\ ${ }^{3}$ Div. of X-ray Physics, Dept. of Physical Sciences, \\ Univ. of Helsinki, Helsinki FI-00014, FI
}

(Dated: October 24, 2018)

\begin{abstract}
Inelastic losses are crucial to a quantitative analysis of x-ray absorption spectra. However, current treatments are semi-phenomenological in nature. Here a first-principles, many-pole generalization of the plasmon-pole model is developed for improved calculations of inelastic losses. The method is based on the GW approximation for the self-energy and real space multiple scattering calculations of the dielectric function for a given system. The model retains the efficiency of the plasmonpole model and is applicable both to periodic and aperiodic materials over a wide energy range. The same many-pole model is applied to extended GW calculations of the quasiparticle spectral function. This yields estimates of multi-electron excitation effects, e.g., the many-body amplitude factor $S_{0}^{2}$ due to intrinsic losses. Illustrative calculations are compared with other GW calculations of the self-energy, the inelastic mean free path, and experimental x-ray absorption spectra.
\end{abstract}




\section{INTRODUCTION}

The theory of the extended x-ray absorption fine structure (EXAFS) is now well developed and can be calculated quantitatively in many systems. [1] However, x-ray absorption near edge structure (XANES) calculations have remained only semi-quantitative at best. [2] One of the reasons for this disparity is a lack of accurate treatments of inelastic losses in the near edge region. For example, traditional calculations of EXAFS typically rely on simplified or semi-phenomenological models of inelastic losses in terms of a complex, energy-dependent exchange-correlation potential, i.e., the quasi-particle self-energy $\Sigma(E)$, where $E$ is the quasiparticle energy. In addition a many body amplitude factor $S_{0}^{2}$ must be applied to the EXAFS signal to account for intrinsic losses, though this is frequently ignored or considered to be a free parameter. 2] Two commonly used models for the self-energy in x-ray absorption spectra (XAS) are i) the Hedin-Lundqvist plasmon-pole model, and ii) the Dirac-Hara exchange approximation plus a constant complex potential. [1, 3, 4, 5, 6] Since the self-energy is smoothly varying at high energy and relatively small compared to the kinetic energy, these approximations are often adequate for EXAFS. However, variations in the self-energy tend to be large in the XANES region, i.e., within the first $50 \mathrm{eV}$ above the Fermi energy, and neither of the above models describes this variation correctly. The energy scale mentioned above is set by the dominant excitations in the system, and is comparable to the mean plasma frequency $\omega_{p}$, which is typically about 10-30 eV. Thus the EXAFS (characterized by weak scattering due to large loss) and XANES (characterized by large scattering and low loss) regions correspond to low and high energy relative to $\omega_{p}$. As a result, the variation in $\Sigma(E)$ with energy leads to significant errors both in amplitude and peak positions in the XANES.

In an effort to improve on these simplified models we present here a many-pole GW approximation for the self-energy, [7, 8, 9] based on real space multiple-scattering calculations of the inverse dielectric function for a given system. Our goal is to develop an approach which can be applied routinely both for EXAFS and XANES. Analogous many-pole models have been used previously in calculations of the self-energy, 10] and of the inelastic mean free path (IMFP), [11, 12] with experimental optical data as input. A few first principles approaches that make use of pole approximations have also been developed.[13, 14, 15] For example Ref. 15 makes use of a band-Lanczos algorithm to calculate a many-pole approxi- 
mation to the inverse dielectric matrix. For reviews of other approaches to GW calculations see Refs. 8 and 16 .

Our many-pole model yields semi-quantitative self-energies over a wide range of photoelectron energies from the near-edge to about $10^{3} \mathrm{eV}$, which is adequate to cover both the XANES and EXAFS regions.[17] The approach has a number of advantages for practical calculations. First the method is computationally efficient in that only a few cpu hours are required to calculate the dielectric function, self-energy, and spectral function for a given system. This is significant since XANES calculations typically take several cpu-hours, while full GW self-energy calculations over the complete energy range of XAS experiments are currently impractical. Finally, the approach is applicable to a wide class of materials including metals, insulators, and molecular systems.

The strategy of our treatment of inelastic losses is as follows. We begin with a first principles calculation of the energy loss spectrum $L(\omega)=-\operatorname{Im}\left[\epsilon(\boldsymbol{q}=0, \omega)^{-1}\right]$ in the long wavelength limit $\boldsymbol{q}=0$. [18, 19] Next this loss function is incorporated into a many-pole model for the self-energy which is an extension of the single plasmon-pole model of Hedin and Lundqvist. [7, 20, 21] This self-energy yields system dependent extrinsic losses due to the lifetime of the quasi-particle over a broad energy range. Next, to account for intrinsic losses, i.e., losses due to excitations of the system in response to the sudden creation of the core hole, we apply our many-pole model to a calculation of the quasiparticle spectral function using an extension of the GW approximation based on the quasi-boson model.[22, 23, 24] This yields corrections to the quasi-particle approximation for XAS in terms of a convolution of the quasi-particle absorption spectrum with the spectral function. Moreover, the approach naturally includes interference terms between extrinsic and intrinsic losses and describes the crossover from the adiabatic- to sudden-approximation limits.

The remainder of this paper is organized as follows. We begin with a brief description of the single plasmon-pole GW model for the self-energy, together with our extension to many poles. We then describe our approach for calculating the dielectric function at zero momentum transfer, as well as the extrapolation to finite momentum transfer. Next we compare our results for the self-energy and the IMFP with other calculations, as well as with experimental results for the IMFP. Subsequently we present our calculation of the quasiparticle spectral function and it's relation to the self-energy. We then compare our calculations of XAFS with experimental as well as theoretical results. Finally, we summarize 
our results and discuss possible improvements.

\section{MANY-POLE SELF-ENERGY}

The many-pole model for the self-energy developed here is an extension of the plasmonpole (PP) model of Hedin and Lundqvist, [7, 20, 21] and contains many of the same ingredients. Thus we begin with a brief description of the PP model, and subsequently describe the extension to a more general loss function used in this work. A more detailed description of the plasmon pole model is given in Appendix . Throughout this paper all quantities are given in Hartree atomic units $\left(e=\hbar=m_{e}=1\right)$ unless otherwise noted. We begin with the GW approximation for the self-energy [7] of a homogeneous electron gas in the momentum representation,

$$
\begin{aligned}
\Sigma(\boldsymbol{k}, E) & =i \int \frac{d^{3} q}{(2 \pi)^{3}} \frac{d \omega}{2 \pi} \frac{V(q)}{\epsilon(\boldsymbol{q}, \omega)} \\
& \times \frac{1}{E-\omega-E_{\boldsymbol{k}-\boldsymbol{q}}+i\left(|\boldsymbol{k}-\boldsymbol{q}|-k_{F}\right) \delta},
\end{aligned}
$$

where $k_{F}$ is the Fermi momentum. In frequency space the imaginary part of the inverse dielectric function (i.e., the loss function of the electron gas) is modeled as a single pole at $\omega(q)=\left[\omega_{p}^{2}+a q^{2}+b q^{4}\right]^{1 / 2}$, where the coefficients of the dispersion $a=k_{F}^{2} / 3$ and $b=1 / 4$ are chosen following the prescriptions of Hedin and Lundqvist. [7, 21] This gives a single-pole model of the inverse dielectric function where

$$
-\operatorname{Im}\left[\epsilon(\boldsymbol{q}, \omega)^{-1}\right]=\pi \omega_{p}^{2} \delta\left[\omega^{2}-\omega(q)^{2}\right]
$$

and

$$
\operatorname{Re}\left[\epsilon(\boldsymbol{q}, \omega)^{-1}\right]=1+\frac{\omega_{p}^{2}}{\omega^{2}-\omega(q)^{2}} .
$$

Inserting these results into Eq. (11) then yields two terms: the first term can be integrated analytically and gives a static Hartree-Fock exchange potential $\Sigma_{H F}$ which, in the local density approximation (LDA) is termed Dirac-Hara exchange,

$$
\Sigma_{H F}(k)=-\frac{k_{F}}{\pi}\left[1+\frac{k_{F}^{2}-k^{2}}{2 k k_{F}} \ln \left|\frac{k_{F}+k}{k_{F}-k}\right|\right] .
$$

The second term, denoted by $\Sigma_{d}\left(\boldsymbol{k}, E ; \omega_{p}\right)$, is the dynamically screened exchange-correlation contribution. This contribution arises from the creation of virtual bosons. The integrals 
over frequency and solid angle can be performed analytically[21], leaving an expression for $\Sigma_{d}\left(\boldsymbol{k}, E ; \omega_{p}\right)$ in terms of a single integral over momentum transfer $\boldsymbol{q}$. This formulation has been used extensively to calculate the mean self-energy $\Sigma(E)$ within the LDA over a broad range of energies for EXAFS spectra.[1] The PP approximation works well at high energies and more generally for systems with sharp plasmon-peaks in the inverse dielectric function (e.g., Al), which can be described by nearly free electron gas models. On the other hand the model often loses accuracy at low energies for transition metals, insulators and molecules with more complex loss spectra, and in practice often gives unphysical structure to the self-energy near $\omega_{p}$. [1]

In order to improve on the plasmon-pole approximation, we now introduce a more realistic representation for the inverse dielectric function, using a sum over discrete poles. This representation preserves the analytical character of $\epsilon(\omega)^{-1}$, and corresponds to a distribution of bosonic excitations describing the dielectric response of a material, including both interband and intraband excitations. The inclusion of this excitation spectrum in the self-energy naturally broadens the single PP model in a way which is characteristic of a given system. Moreover, the representation can be systematically improved.

Two steps must be accomplished in order to extend the PP self-energy to a many-pole self-energy (MPSE): i) The first step is to obtain a suitable approximation to the energy loss function $L(\omega)=-\operatorname{Im}\left[\epsilon(\boldsymbol{q}=0, \omega)^{-1}\right]$. This can be done either by calculation, as is done here, or from experimental optical constants. ii) The second step is to extend the $\boldsymbol{q}=0$ result to finite momentum transfer, by representing it as a weighted sum of poles, each of the form Eq. (2), which together conserve the overall strength. In addition we approximate the single particle Green's function $G(E)$ as that for a free particle. This is the first term in the multiple-scattering expansion and ignores fine structure; hence the calculated selfenergy represents a uniform average. With these conditions the net self-energy is simply the Hartree-Fock exchange contribution plus a dynamically screened exchange-correlation contribution which is given by a weighted sum of single pole terms,

$$
\Sigma_{d}(k, E)=\sum_{i} g_{i} \Sigma_{d}\left(k, E ; \omega_{i}\right)
$$

with weights $g_{i}$ and plasma frequencies $\omega_{i}$. As mentioned above, $\Sigma_{d}(k, E)$ is given by a single integral over momentum transfer $|\boldsymbol{q}|$, making the calculation quite efficient. Fig. 口illustrates the self energy from our many pole model for $\mathrm{Cu}$. Note that only 20 poles were needed to 


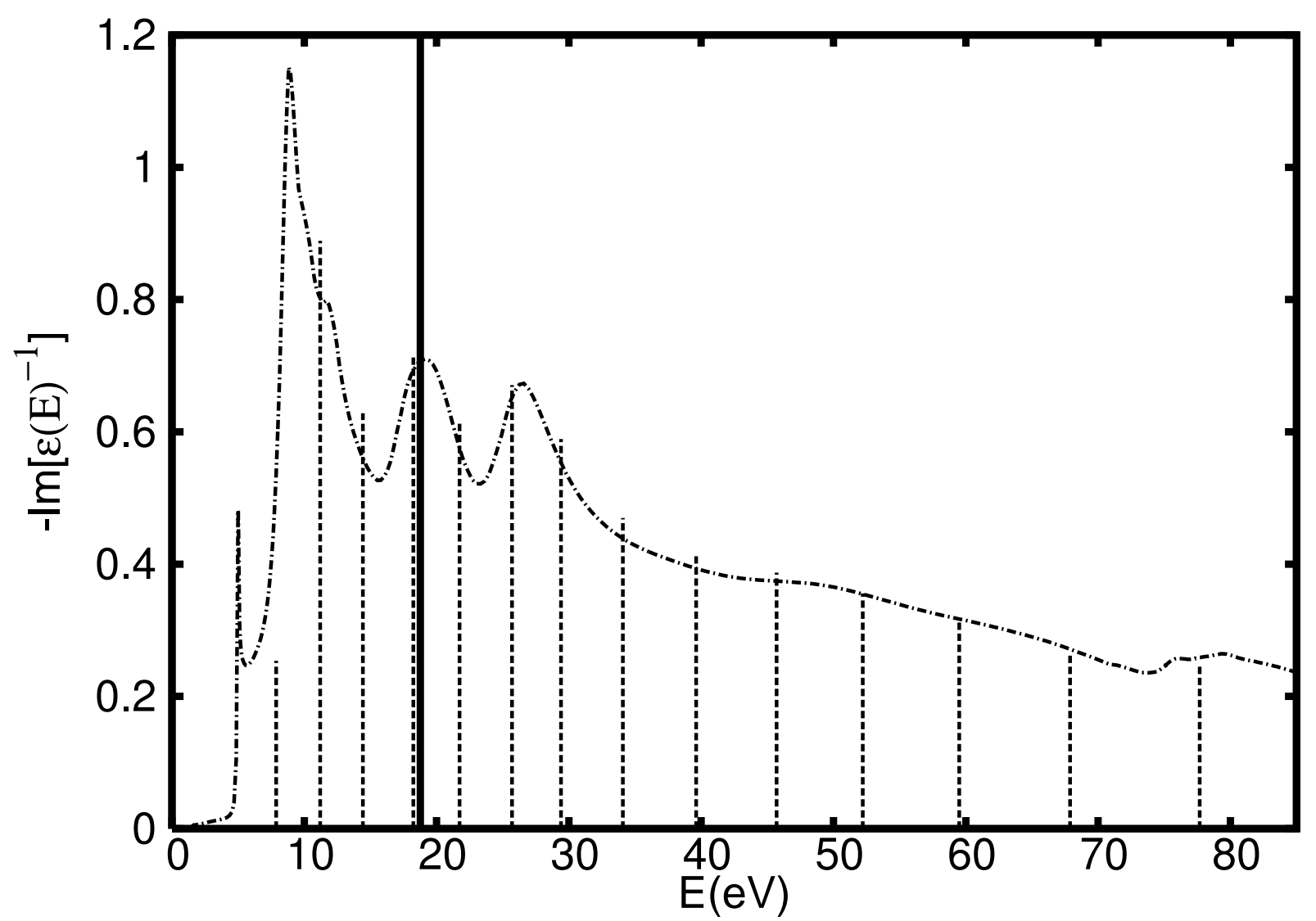

FIG. 1: Energy loss function $L(\omega)=-\operatorname{Im}\left[\epsilon(\omega)^{-1}\right]$ in the long wavelength limit for Cu modeled either as a single pole (solid vertical line), or as a sum of weighted poles (vertical dashes), compared to the loss function as calculated by the FEFF8 code (dot dashed).

converge this calculation, despite the relatively broad loss function of $\mathrm{Cu}$.

\section{Inverse dielectric function}

In our approach the inverse dielectric function is calculated using the real-space Green's function (RSGF) method as follows: First, a modification to the RSGF code FEFF8[25, 26] is used to calculate the total absorption cross section $\sigma(\omega)$ for a given material over a broad spectrum, by summing the contributions from all occupied initial states. [4, 19] The results presented in this paper make use of atomic initial states. However current developments allow for the description of a continuous band of initial states within the FEFF real space MS framework, [18] which may further improve the results. The imaginary part of the dielectric function $\epsilon_{2}$ is directly related to the total absorption cross section per atom $\sigma(\omega)$ as calculated 


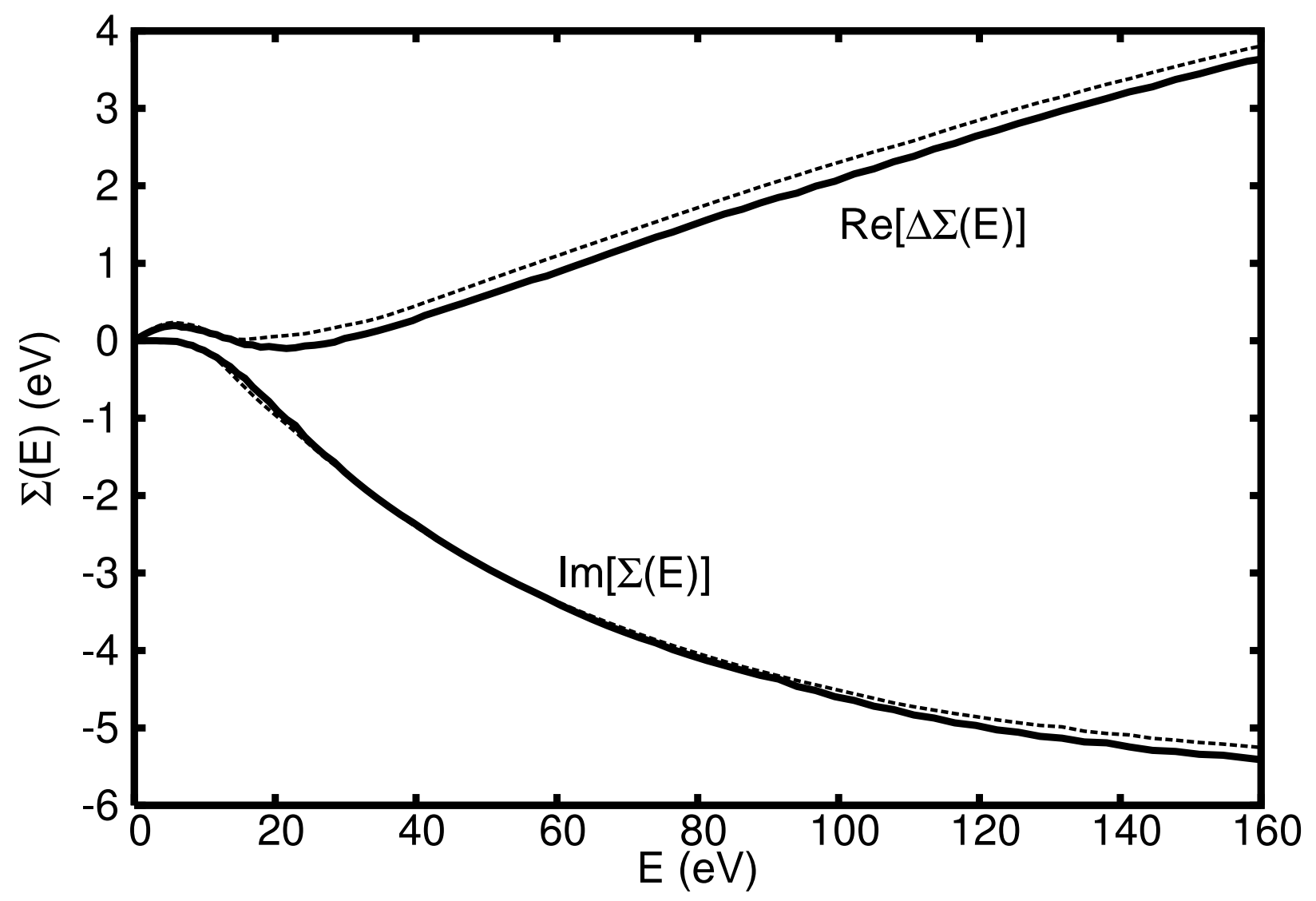

FIG. 2: Quasiparticle self-energy for Cu calculated using a loss function with fine structure (dashes) or without fine structure (solid).

by the FEFF code. $\epsilon_{2}=(n / \alpha \omega) \sigma(\omega)$ where $n$ is the atomic number density and $\alpha$ is the fine structure constant. The real part $\epsilon_{1}(\omega)$ is then obtained via a Kramers-Kronig transform, and finally $L(\omega)=-\operatorname{Im}\left[\epsilon(\omega)^{-1}\right]$ is formed by inverting $\epsilon(\omega)$. This could be a computationally demanding operation. However, because the self-energy involves an integral over $\epsilon(\omega)^{-1}$, the fine structure can be neglected in all but the lowest energy part of the dielectric function (i.e., the first $20 \mathrm{eV}$ ), as shown in Fig. 2. This approximation considerably reduces the computational effort. It should be noted that this prescription for the calculation of the loss function also neglects local field effects due to the off diagonal components of the dielectric matrix. Nevertheless, the method has been shown to give reasonable agreement with experiment for a variety of materials.[18, 19] Moreover, neither the self-energy nor the absorption spectrum (i.e. EXAFS and XANES) are highly sensitive to details of the loss function provided the overall weight is conserved since these quantities are given by integrals over the loss function. 


\section{Extension to finite momentum transfer}

In order to extend the inverse dielectric function to finite momentum transfer $q$, we represent the imaginary part of the loss function as a sum of closely-spaced delta functions

$$
L(\boldsymbol{q}, \omega)=-\operatorname{Im}\left[\epsilon(\boldsymbol{q}, \omega)^{-1}\right]=\pi \sum_{i} g_{i} \omega_{i}^{2} \delta\left(\omega^{2}-\omega_{i}(q)^{2}\right) .
$$

Typically of order $10^{1}-10^{2}$ poles are sufficient. Matching the many-pole model $\epsilon(\boldsymbol{q}, \omega)^{-1}$ evaluated at zero momentum transfer to the calculation of $\epsilon(\omega)^{-1}$ then gives the weights $g_{i}$ and pole locations $\omega_{i}$ respectively. Our prescription for this match is as follows: First, the loss function is split into $N$ regions, each of which is represented by a single pole. For each region of width $\Delta_{i}$, the pole strength and position are chosen to preserve first and first-inverse moments, yielding the equations defining $g_{i}$ and $\omega_{i}$

$$
\begin{aligned}
g_{i} \omega_{i}^{2} & =-\frac{2}{\pi} \int_{\Delta_{i}} d \omega \omega \operatorname{Im}\left[\epsilon(\omega)^{-1}\right] \\
g_{i} & =-\frac{2}{\pi} \int_{\Delta_{i}} \frac{d \omega}{\omega} \operatorname{Im}\left[\epsilon(\omega)^{-1}\right] .
\end{aligned}
$$

For simplicity we also use the same plasmon dispersion as in the PP model. This approximation has been checked against a dispersion relation which maintains the width of the pole at high momentum transfers and gives similar results for materials with a broad loss function such as $\mathrm{Cu}, \mathrm{Ag}$, and Diamond. For materials with a sharp loss function (i.e. Al, $\mathrm{Si}$, and $\mathrm{Na}$ ) this approximation may not be adequate at low energies (below the plasmon energy) where the contribution from the particle hole continuum can dominate the loss.

Finally, for very low energies (i.e., the first few eV where our multiple-scattering calculations are least reliable) the calculated loss function must be corrected. For metals a Drude term is added and otherwise a uniform shift of the frequencies $\left\{\omega_{i}\right\}$ is carried out while scaling the resultant poles, so that the inverse moment matches either empirical values or accurate calculations of the static dielectric constant $\epsilon(0)$, while leaving the first moment unchanged.

For stability we have found it important to preserve the inverse first frequency moment, since this ensures cancellation of the logarithmic singularity in the derivative of $\Sigma_{H F}$ at $k=k_{F}$ and $E=E_{F}$. [20] This singular behavior otherwise shows up as a sharp rise in $\operatorname{Re}[\Sigma(\boldsymbol{k}(E), E)]$ within the first $10-20 \mathrm{eV}$ above $E_{F}$. In metals, where cancellation is perfect, $\operatorname{Re}[\Sigma(\boldsymbol{k}(E), E)]$ is fairly flat near $\mathrm{E}=E_{F}$. In insulators, however, this singular behavior is 
found to enhance the jump in $\operatorname{Re}\left[\Sigma\left(\boldsymbol{k}\left(E_{F}\right), E_{F}\right)\right]$. Thus our prescription requires a separate estimate either of the static dielectric function $\epsilon(0)$ for the case of insulators or the Drude parameters for metals and semi-metals. These quantities have been used previously to parameterize the dielectric matrix, [13, 27, 28, 29, 30, 31] for example Ref. 27 uses similar parameters to modify a single pole model of the dielectric function, while Refs. 28 and 29 generalize to a full dielectric matrix.

Our self-energy model is similar to those of Penn[11] and Horsch et al.[10] for the valence contribution. One difference is that our formulation neglects the relatively small particle hole continuum contributions below the plasmon onset. Another is that our formulation includes a first order correction to the quasipartle energy as well as a renormalization constant $Z$ which accounts for the quasiparticle spectral weight. Appendix ${ }_{\mathrm{a}}$ gives a short discussion of the equivalence of the formulas for the self-energy given by Quinn (which is the starting point for Penn's calculations) and by Lunqvist. A further note must be made regarding the difference between our many-pole model and the LDA implementation in the FEFF8.2 code. The plasmon frequency in the current LDA model in FEFF8.2 is dependent on the electron density as a function of spacial coordinates. In the model discussed here, adding spacial dependence greatly complicates the theory and is therefore ignored. Thus our approach gives the spacially averaged quasiparticle correction for the whole system. We have found that in the XANES region, the self-energy effects on the spectrum are not sensitive to the density dependence. Also our calculations use the interstitial density to determine the Fermi momentum; the interstitial density was chosen, instead of the average density, because we want the model to capture the behavior of the self-energy due to interaction with the valence electrons. For the core electrons FEFF8 already has an option to use a non-local Dirac-Fock exchange which can be applied with our many-pole model.[32]

\section{EXTRINSIC LOSSES}

In this section we present results which characterize the "extrinsic losses," in XAS, namely the self-energy and the inelastic electron mean-free-path. To confirm that our approach gives improved results when compared to the PP model, we have compared with other calculations of the self energy, including the single PP model of Hedin and Lundqvist and a more accurate many-pole approximation. [15, 33] Fig. 3 shows our many-pole self-energy 

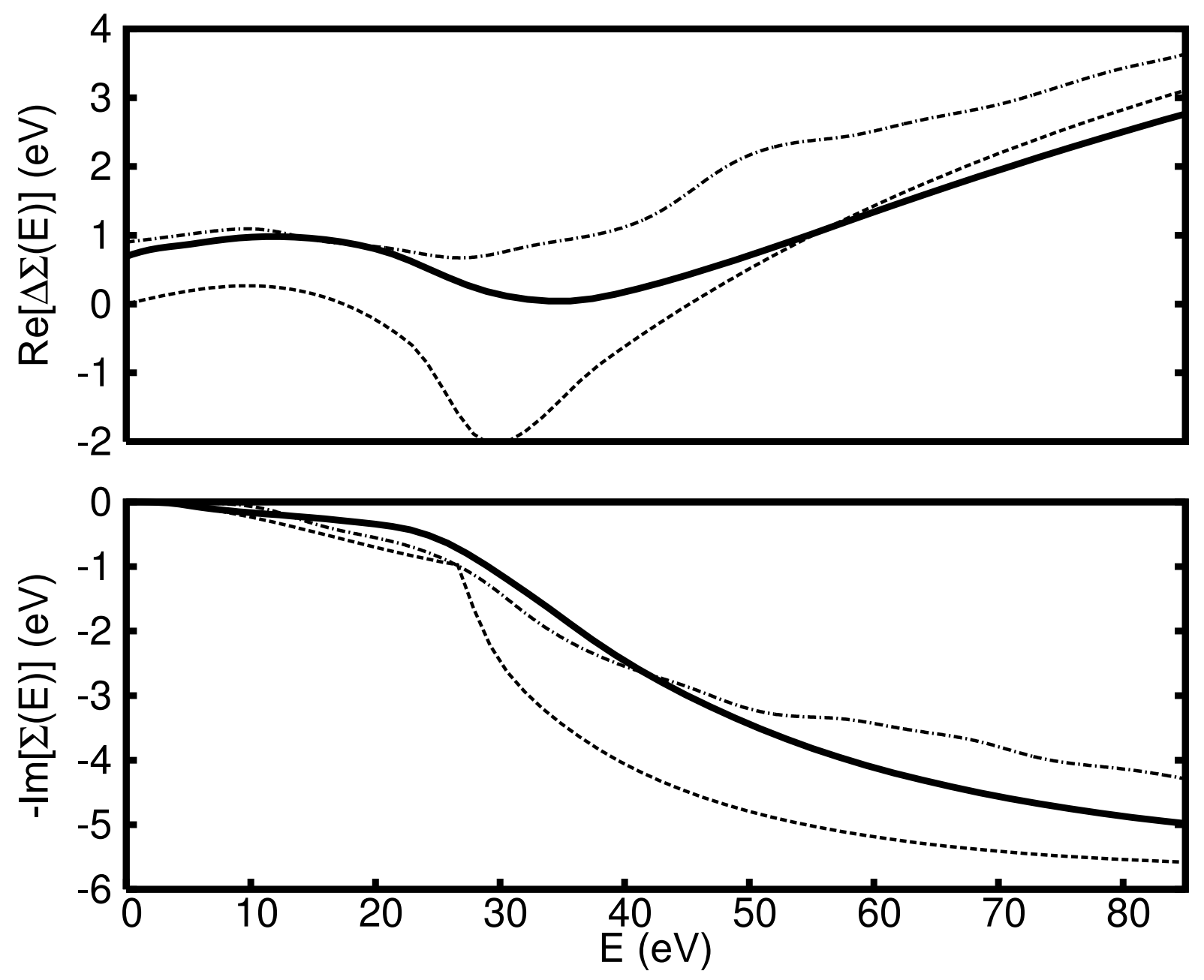

FIG. 3: a) Real (top) and b) imaginary (bottom) parts of the self-energy for Diamond calculated using our many-pole model (solid); the Hedin-Lundqvist PP model (dashes); and the iterative band-Lanczos calculation of Refs. 15 and 33 (dot dash).

for diamond compared with the single-pole model as well as the band-Lanczos calculation of Refs. 15 and 33. In addition, we use our results to calculate the electron inelastic mean free path (IMFP).

$$
\lambda(E)=\sqrt{\frac{E}{2}} \frac{1}{|\operatorname{Im}[\Sigma(E)]|} .
$$

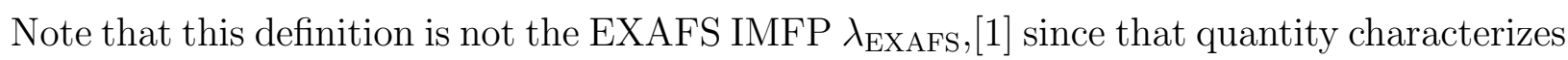
the decay of the EXAFS amplitude and includes both core hole broadening $\Gamma$ and the self-energy, $\left.\lambda_{\mathrm{EXAFS}}=(2 E)^{(1 / 2)} /[\mid \operatorname{Im} \Sigma(E)] \mid+\Gamma / 2\right]$. Fig. 4 shows our results for the IMFP for Mo, and for comparison the single-pole model, an optical model which uses the Penn algorithm, [11, 12] and experiment. [34] Other applications of our many-pole model as well as 
IMFP results for a number of materials have recently been presented by Sorini et al. [17] As can be seen in Fig. . our self-energy gives improved results for the IMFP over a broad range of energies.

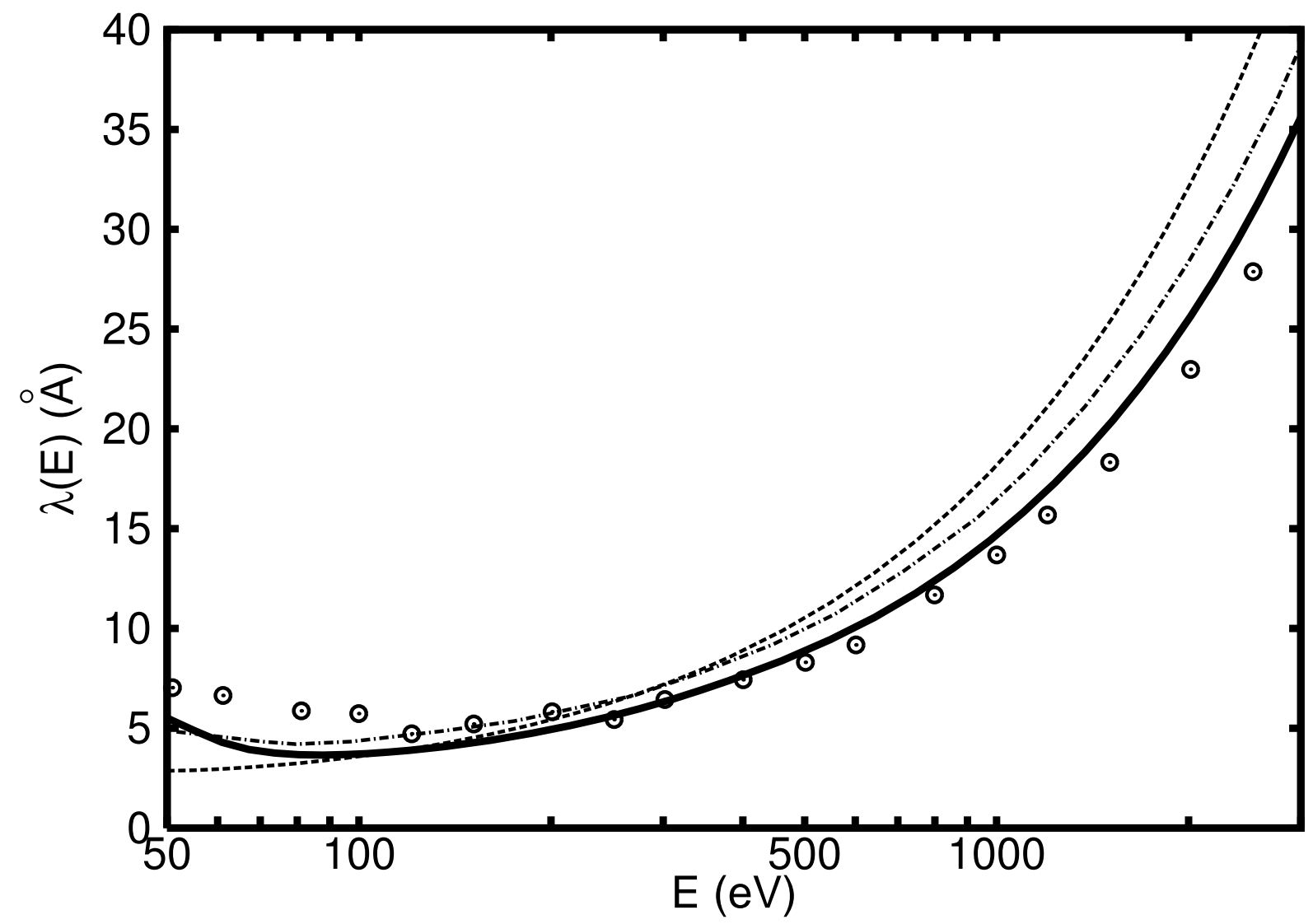

FIG. 4: Inelastic mean free path for Mo calculated using our many pole model (solid); the HedinLundqvist single pole model (dashes); a many-pole model based on optical data [11, 12, 34] (dot dash); and experimental data from Tanuma et al. [34] (circles)

\section{INTRINSIC LOSSES}

In this section we describe the treatment of intrinsic losses in a system in terms of an effective quasi-particle "spectral function." [22] The many-pole GW self-energy developed above is adequate to describe the extrinsic losses of the photoelectron in the independent particle (i.e., quasi-particle) approximation for the XAS. However this approximation neglects intrinsic losses due to the excitations in the absorbing medium that arise from the sudden creation of the core-hole. As a consequence of these excitations, the energy of the 
absorbing photoelectron is lowered, resulting in a shift in the absorption signal. Moreover, one must also take into account interference between the intrinsic and extrinsic losses. Both intrinsic losses and interference terms can be accounted for in terms of an energy-dependent spectral function.

Here we implement a many-pole model for the spectral function derived from a direct extension to the GW approximation and based on a quasi-boson model. [22] Within the approximations detailed in Ref. 22, the full many-body spectrum is given by a convolution of the single quasi-particle spectrum with an energy dependent spectral function $A_{\text {eff }}\left(\omega, \omega^{\prime}\right)$, i.e.,

$$
\mu(\omega)=\int d \omega^{\prime} A_{\mathrm{eff}}\left(\omega, \omega^{\prime}\right) \mu_{\mathrm{qp}}\left(\omega-\omega^{\prime}\right)
$$

Here $A_{\text {eff }}\left(\omega, \omega^{\prime}\right)$ characterizes the probability density that a photon excites a photoelectron of energy $\omega-\omega^{\prime}$, as well as additional excitations (e.g., plasmons, electron-hole pairs, etc.) with energy $\omega^{\prime}$.

Similarly the intrinsic many-body corrections to the EXAFS $\chi$ can be represented by a convolution of the single quasi-particle signal $\chi_{\mathrm{qp}}$ and the normalized effective spectral function $A_{\text {eff }}\left(\omega, \omega^{\prime}\right)[22]$

$$
\chi(\omega)=\int d \omega^{\prime} A_{\mathrm{eff}}\left(\omega, \omega^{\prime}\right) \chi_{\mathrm{qp}}\left(\omega-\omega^{\prime}\right)
$$

The convolution in Eq. (11) leads to a path dependent amplitude reduction in the EXAFS signal $S_{0, j}^{2}$. Since the EXAFS $\chi$ can be expressed as a sum of rapidly varying sinusoidal contributions from each photoelectron scattering path[35] with smooth amplitudes $\chi_{j}(\omega) \propto$ $\exp \left(2 i R_{j} k(\omega)\right)$, the amplitude reduction for each path is given by a phasor-summation

$$
S_{0, j}^{2}(\omega) \approx \int d \omega^{\prime} A_{\mathrm{eff}}\left(\omega, \omega^{\prime}\right) e^{2 i R_{j}\left[k\left(\omega-\omega^{\prime}\right)-k(\omega)\right]}
$$

where $R_{j}$ is one half the total scattering path length of the photoelectron. As $S_{0, j}^{2}(\omega)$ is only weakly energy dependent, this amplitude factor can usually be approximated by a constant over a broad range of energies, consistent with experimental observation. In contrast the behavior of $S_{0, j}^{2}(\omega)$ for the single-pole model exhibits much more variation.

The spectral function can be considered to be made up of a quasiparticle peak and satellites. Since broadening can be added separately, the quasiparticle part can be represented as a delta function of net magnitude $Z_{\text {eff }}$ at zero excitation energy, while the satellites 


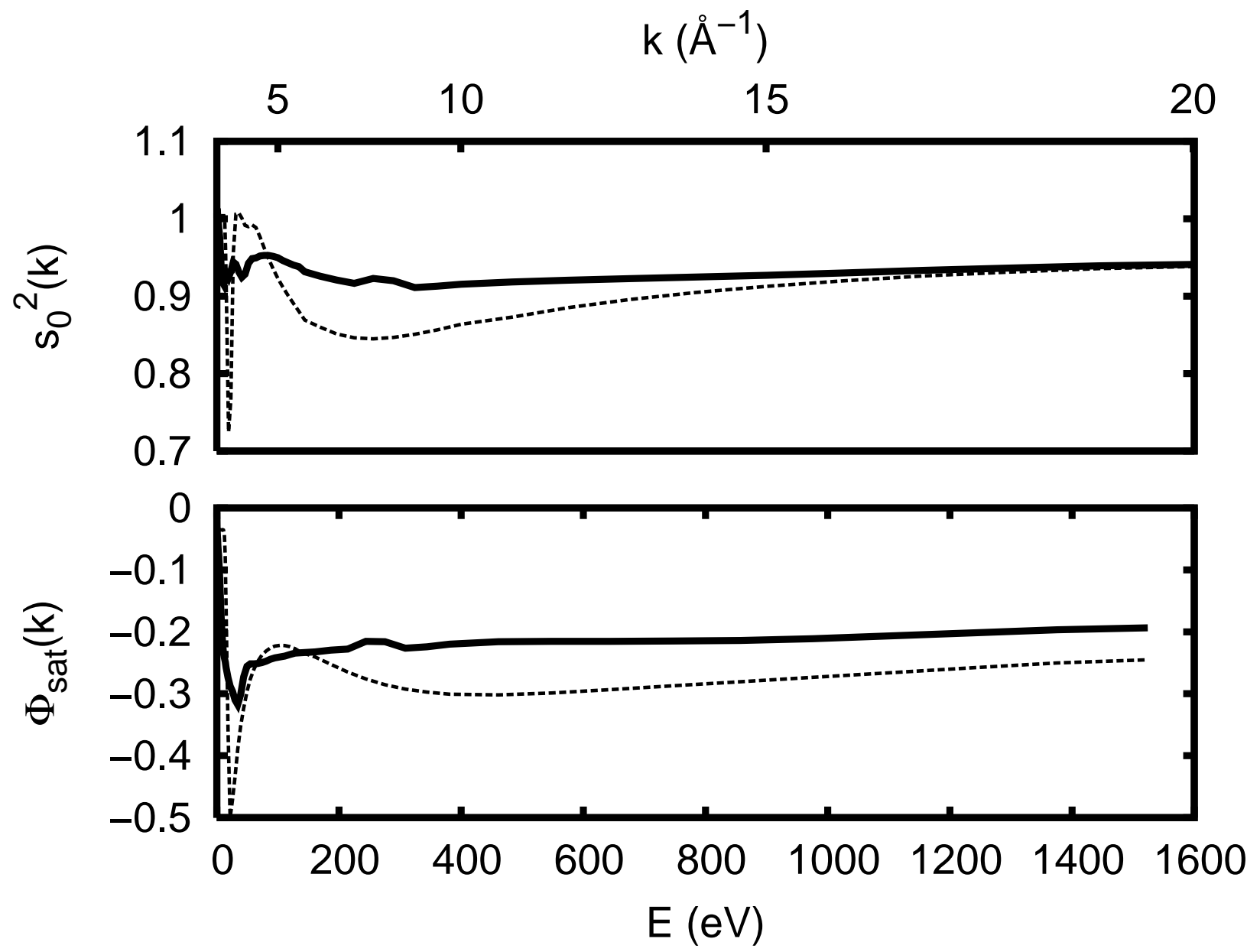

FIG. 5: EXAFS $S_{0}^{2}(k)$ (upper) and the net phase $\Phi_{\text {sat }}$ of $A_{\text {sat }}(k)$ (lower) for our many-pole model (solid) and the Hedin-Lundqvist single pole model (dashes). Note that the many-body amplitude and phase factors are approximately constant over a broad range of energies in the EXAFS $(\approx 200$ $\mathrm{eV}$ and above) with $S_{0}^{2}$ ranging from $\approx 0.91-0.94$ and $\Phi_{\text {sat }}$ in the range -0.21 to -0.18 rad.

represent contributions from inelastic excitations in the medium. Within the quasi-boson approximation [22] one has

$$
A_{\mathrm{eff}}\left(\omega, \omega^{\prime}\right)=N(\omega)\left[[1+2 a(\omega)] \delta\left(\omega^{\prime}\right)+A^{\mathrm{sat}}\left(\omega, \omega^{\prime}\right)\right]
$$

where $N(\omega)$ is a normalization constant which preserves the overall spectral weight at each $\omega^{\prime}$. In our approach the satellite contribution is further broken down into three terms corresponding to the origin of the inelastic excitation; an extrinsic part $A_{\text {ext }}^{\text {sat }}$ coming from excitations created by the photoelectron, an intrinsic part $A_{\mathrm{intr}}^{\mathrm{sat}}$ arising from the excitations created by the sudden appearance of the core hole, and a term $A_{\text {inter }}^{\text {sat }}$ from the interference 


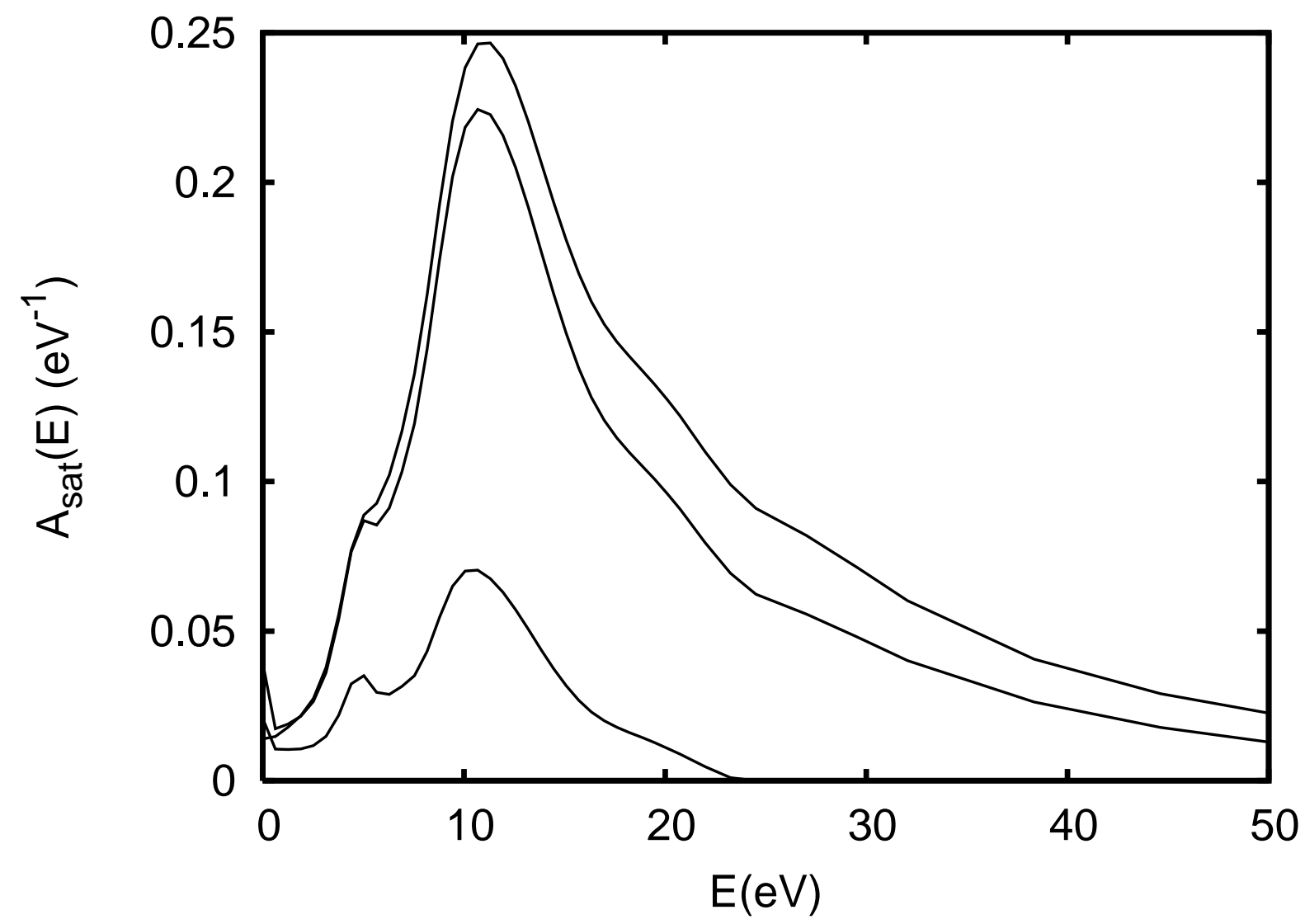

FIG. 6: Satellite spectral function for a range of photoelectron momenta. From top to bottom, $k=16,8$, and $4 \AA^{-1}$.

between them

$$
A^{\mathrm{sat}}\left(\omega, \omega^{\prime}\right)=A_{\mathrm{ext}}^{\mathrm{sat}}\left(\omega, \omega^{\prime}\right)+A_{\mathrm{intr}}^{\mathrm{sat}}\left(\omega, \omega^{\prime}\right)-2 A_{\mathrm{inter}}^{\mathrm{sat}}\left(\omega, \omega^{\prime}\right) .
$$

The effect of the interference tends to reduce the satellite part of the spectral function, and the spectral weight is shifted back to the quasiparticle peak. This variation accounts for the $a(\omega)$ factor appearing in the weight of the quasiparticle peak. The detailed derivation of the components of the spectral function arising from a PP dielectric function have been presented elsewhere. [22] Here it is sufficient to present results characterized by the extension to a many-pole loss-function as in Eq. (66). Thus the intrinsic and interference contributions 
are given by

$$
\begin{aligned}
A_{\mathrm{intr}}^{\mathrm{sat}}\left(\omega, \omega^{\prime}\right)= & \frac{1}{\pi} \sum_{i} g_{i} \omega_{i}^{2} \int_{0}^{\infty} \frac{d q}{\omega_{i}(q)^{3}} \delta\left(\omega^{\prime}-\omega_{i}(q)\right), \\
A_{\mathrm{inter}}^{\mathrm{sat}}\left(\omega, \omega^{\prime}\right)= & \frac{1}{2 \pi k} \sum_{i} g_{i} \omega_{i}^{2} \int_{0}^{\infty} \frac{d q}{q \omega_{i}(q)^{2}} \delta\left(\omega^{\prime}-\omega_{i}(q)\right) \\
& \times \ln \left[\frac{\omega_{i}(q)-q^{2} / 2+k q}{\omega_{i}(q)-q^{2} / 2-k q}\right], \\
a(\omega)= & \frac{1}{2 \pi k_{0}} \sum_{i} g_{i} \omega_{i}^{2} \int_{0}^{\infty} \frac{d q}{q \omega_{i}(q)^{2}} \\
& \times \ln \left[\frac{\omega_{i}(q)+q^{2} / 2+k_{0} q}{\omega_{i}(q)+q^{2} / 2-k_{0} q}\right]
\end{aligned}
$$

where $k=\left[2\left(\omega-\omega^{\prime}\right)\right]^{1 / 2}$ is the photoelectron wavenumber and $k_{0}=[2(\omega)]^{1 / 2}$ is the on-shell photoelectron wavenumber. The extrinsic contribution to the spectral function can be found from the photoelectron self-energy $\Sigma\left(k, \omega+\omega^{\prime}\right)$ and renormalization constant $Z_{k}$.

$$
\begin{aligned}
A_{\mathrm{ext}}^{\mathrm{sat}}\left(\omega, \omega^{\prime}\right) \approx-\frac{1}{\pi\left|Z_{k_{0}}\right|} & {\left[\frac{\Gamma_{k}+\operatorname{Im} \Sigma\left(k, \omega+\omega^{\prime}\right)}{\left[\omega^{\prime}+\Delta_{k}\right]^{2}+\left[\Gamma_{k}\right]^{2}}\right.} \\
- & \left.\frac{\operatorname{Im} Z_{k_{0}}}{\omega^{\prime}} e^{-\left(\omega^{\prime} / 2 \omega_{p}\right)^{2}}\right],
\end{aligned}
$$

where

$$
\begin{aligned}
& \Delta_{k}=\operatorname{Re}\left[\Sigma\left(k_{0}, \omega\right)-\Sigma\left(k, \omega+\omega^{\prime}\right)\right] \\
& \Gamma_{k}=-\operatorname{Im}\left[\Sigma\left(k_{0}, \omega\right)-\Sigma\left(k, \omega+\omega^{\prime}\right)\right] .
\end{aligned}
$$

\section{XAS CALCULATIONS}

As illustrations of our approach, we now compare our results for the XANES spectra of $\mathrm{Cu}$ and diamond as calculated with the many-pole model against those calculated with the single-pole model, and with experiment. Calculations of the self-energy and the spectral function were converged with respect to the number and distribution of poles used to represent the dielectric function. We find that typically only $10-20$ poles are needed to represent a relatively broad loss function such as that for $\mathrm{Cu}$. The full multiple-scattering FEFF8 calculation for $\mathrm{Cu}$ was converged with respect to the cluster size as well as the angular momentum cutoff $l_{\max }$. There are only two free parameters in our calculations; a small imaginary shift in the potential was used to account for experimental broadening; and a real 
energy shift was introduced to correct for the inaccuracy in Fermi energies calculated by the FEFF8 code.[36]

Comparison with experiment in Fig. 7 and 8 shows a clear improvement both in the phases and amplitudes of the XAFS signal and the near-edge structure. These improvements can be linked respectively, to the real and imaginary parts of the self-energy. The real part induces phase shifts in the signal while the imaginary part is directly related to the inelastic mean free path and hence the amplitudes. Fig. 7 shows our $\mathrm{Cu}$ K edge XANES calculations with both single and many-pole self-energies compared to experiment. A large (500 atom) cluster was used to calculate the spectra up to $\approx 35 \mathrm{eV}$ above the Fermi level, above which a smaller (177 atom) cluster was used with higher angular momentum components. Thus we used $l_{\text {max }}=3$ for low energies and $l_{\max }=4$ above $\approx 35 \mathrm{eV}$. This was done in order to ensure that errors due to finite cluster size and angular momentum cutoff were small compared to effects of the self-energy on the XANES spectrum. The result shows improvement in the amplitudes and phases of the peaks, especially in the region from $\approx 10-50 \mathrm{eV}$ (top). The amplitude of the "whiteline" peak (a) is substantially reduced by the corrected self-energy, while the second peak (b) acquires a phase shift. The dip seen at $\approx 32 \mathrm{eV}$ (c) also attains a significant phase shift and an increase in amplitude. The considerable improvements seen in this low energy XANES region can be attributed to the fact that the plasmon pole self-energy has singular behavior near the plasma frequency. This behavior is absent in the many-pole self-energy which is naturally broadened by the width of the loss function. Furthermore, there is improvement even in the EXAFS region $45-80 \mathrm{eV}$ (bottom). Here the single plasmon pole model gives a smooth, almost featureless curve, whereas the manypole model as well as the experiment show noticeable features at $62 \mathrm{eV}$ (d) and $71 \mathrm{eV}$ (e). Fig. 8 presents similar calculations for the diamond K edge XANES compared to data from non-resonant inelastic x-ray scattering[38]. For diamond we could not fully converge the multiple scattering calculations with respect to cluster size at all energies because of memory requirements of the code. Thus we present our results for a 500 atom cluster with $l_{\max }=2$. Here the results are more difficult to interpret because of errors due to finite cluster size and our approximate treatment of core hole effects. However, qualitative improvement is seen in the amplitudes of the EXAFS from $\approx 25 \mathrm{eV}$ on. Specifically, the feature seen in the experiment at approximately $32 \mathrm{eV}$ (a) is absent in the single plasmon pole calculation, but appears in the new calculation. Also, the three subsequent peaks (b, c, and d) are 

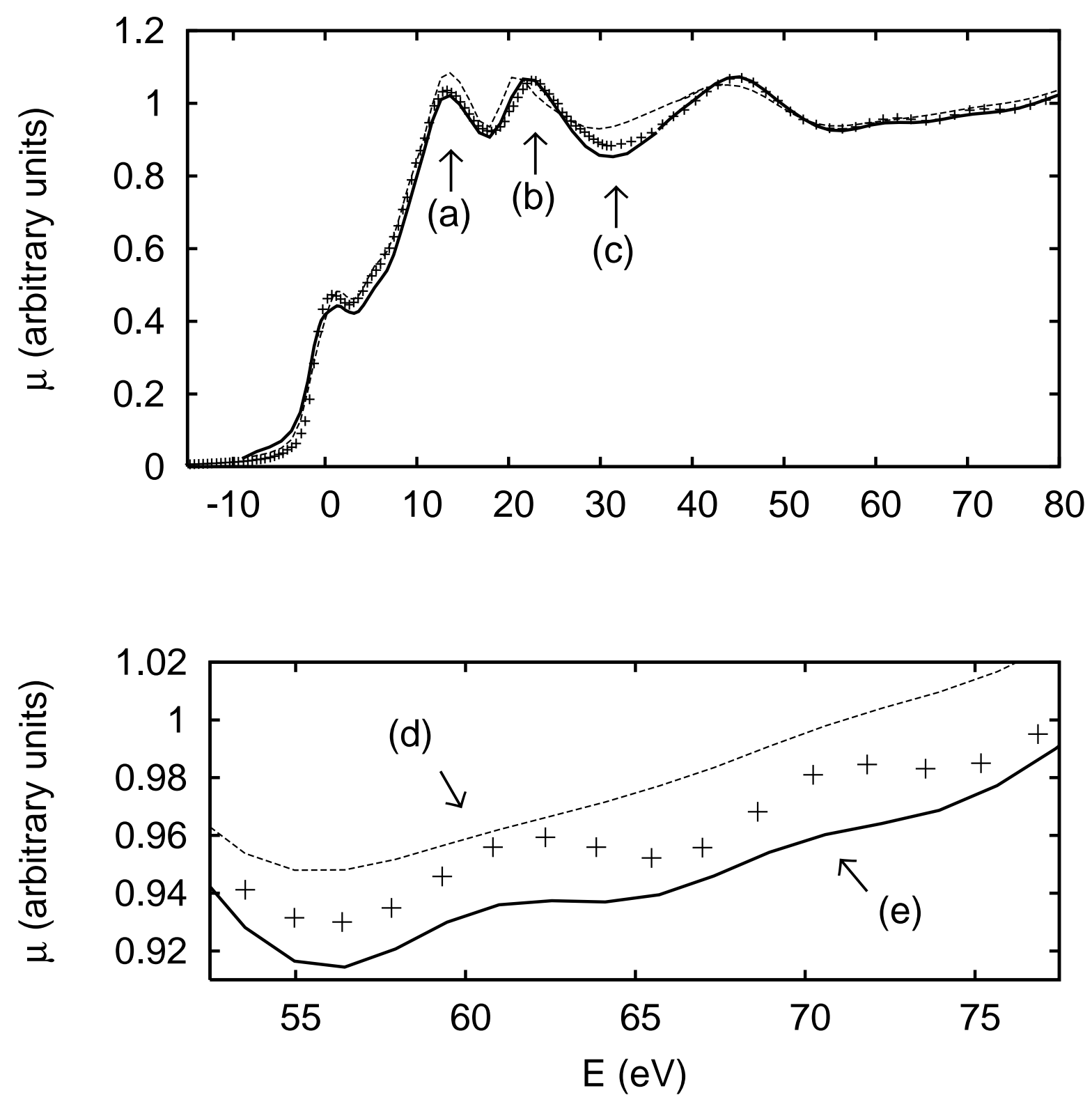

FIG. 7: a) Top: $\mathrm{Cu} \mathrm{K}$ edge XANES calculated from the many-pole self-energy and spectral function of this work (solid), and for comparison the conventional single-pole model (dashes), and experiment [37] (+); b) Bottom: $\mathrm{Cu} \mathrm{K}$ edge EXAFS shown from $k=3.75-4.55 \AA^{-1}$.

enhanced as a result of the new many-pole calculation, giving better qualitative agreement with experiment. To reiterate, the single plasmon pole self-energy has a sharp turn-on of the imaginary part which saturates too early, giving excessive broadening in the range beyond the plasmon energy. Similar self-energy effects have been seen in the F K-edge spectrum of 
LiF, where a more computationally demanding full-GW calculation was performed. [33]

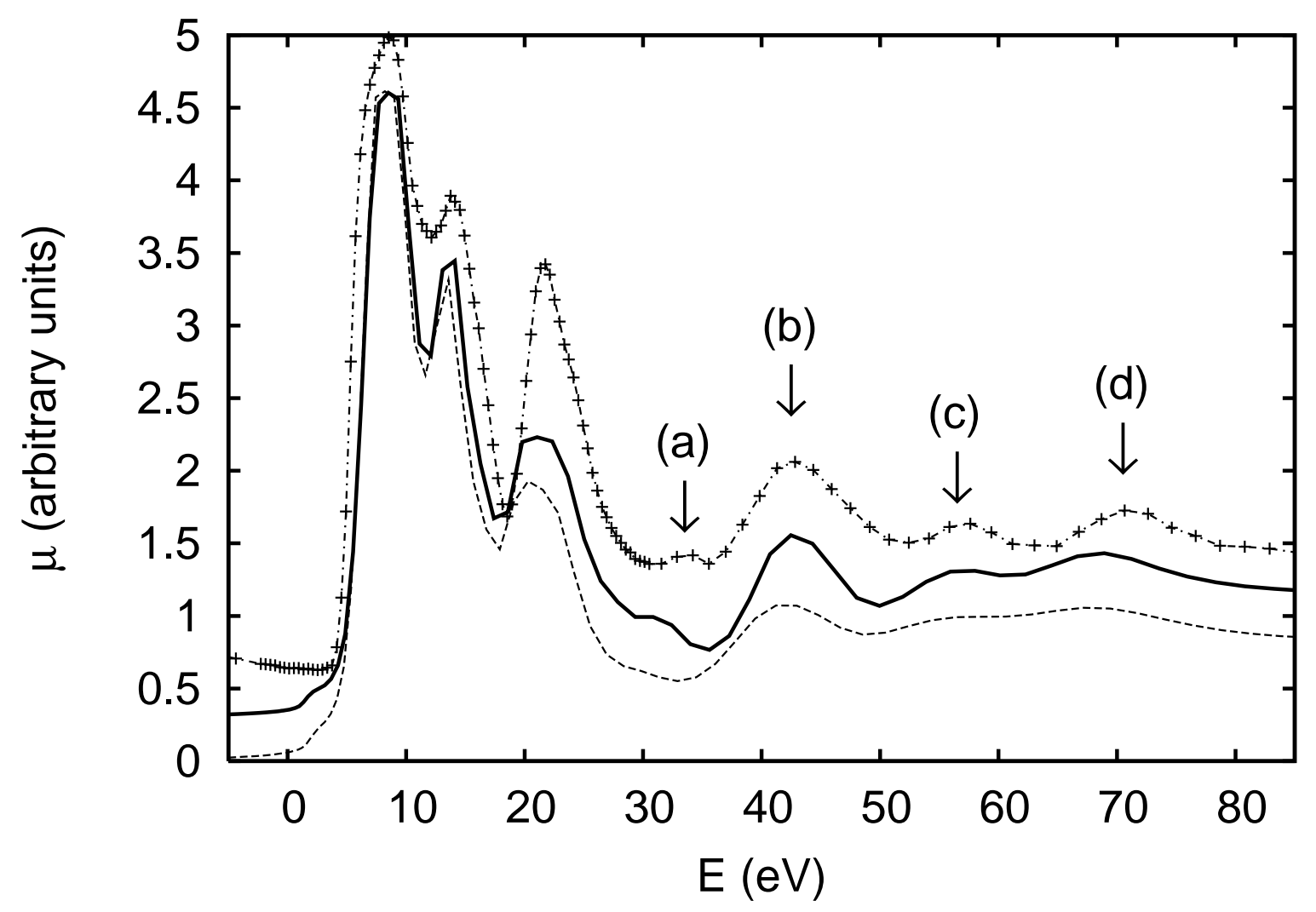

FIG. 8: Diamond K edge XANES calculated with the many-pole self-energy and spectral function of this work (solid), and for comparison the conventional single-pole model (dashes), and experiment $(+) \cdot[38]$

In addition to our XANES calculations, we have performed a comparison of experiment and theory of the $\mathrm{Cu} \mathrm{K}$ edge EXAFS using the analysis software ATHENA.[39] To reduce Debye Waller effects, which are highly correlated with the effects of the self-energy and many-body amplitude reduction factor, we used data taken at a low temperature of $10 \mathrm{~K}$. In order to give a fair comparison of the two theories (PP self-energy and MPSE) we have fixed all parameters to empirical or theoretical values. First the theory and experiment were aligned by matching features in the range $0-300 \mathrm{eV}$. Then background subtraction and normalization was performed using the same spline fitting range as well as normalization range for experiment and theory. The EXAFS $\chi(k)$ was Fourier transformed using a $k$ range of $2.632 \AA^{-1}$ to $15.5 \AA^{-1}$ with a weighting of $k^{1}$. Debye waller factors were set using 


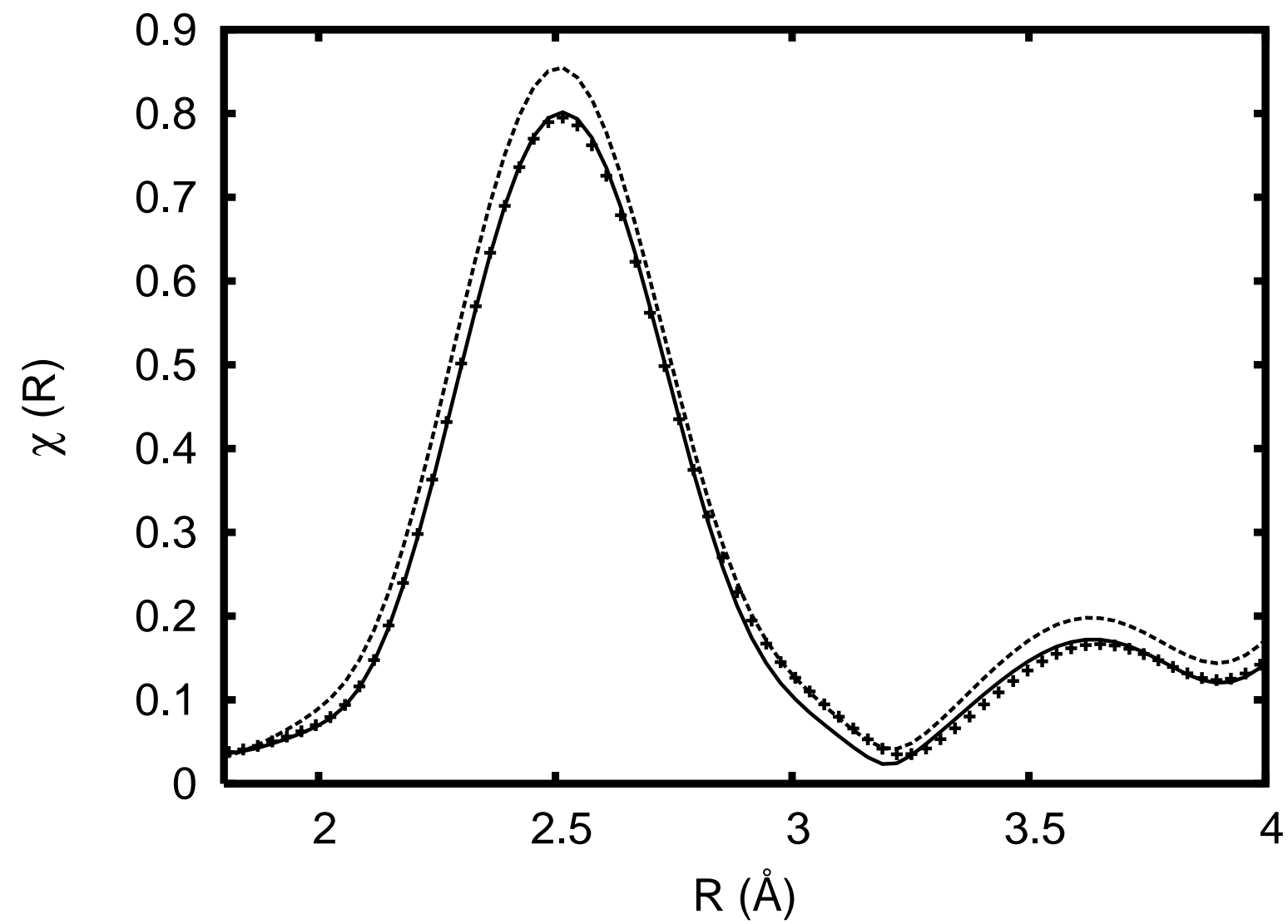

FIG. 9: Comparison of $\mathrm{Cu} \mathrm{K}$ edge EXAFS Fourier transform $\chi(R)$. Theoretical calculations using the many-pole self-energy (solid), the plasmon-pole model (dashed), and Experiment[37] (+). The value of $S_{0}^{2}$ was found to be $\approx 0.93$ over most of the EXAFS range.

the correlated Debye model with $\Theta_{D}=315 \mathrm{~K}$. In addition, the theory was broadened by $0.45 \mathrm{eV}$ half width half max to account for experimental broadening. The estimate of the experimental resolution was obtained by comparing to the width of the edge step. As can be seen in Fig. 9 the amplitude of the first shell peak is reduced by our new treatment of inelastic losses, thus improving the agreement with experiment and demonstrating the adequacy of our calculation of $S_{0}^{2}$. Our value for $S_{0}^{2}(\approx 0.93)$ also agrees with a crude approximation (previously implemented in the FEFF8 code) which calculates the many-body overlap of the atomic system and gives $S_{0}^{2}=0.95$. 


\section{CONCLUSIONS}

An efficient many-pole model for calculations of inelastic losses has been developed and successfully implemented in an extension of the multiple scattering code FEFF8. Our manypole model is based on an ab initio calculation of the zero momentum transfer loss function by means of the RSGF approach implemented in an extension of the FEFF8 code. Extrapolation to finite momentum transfer is performed by representing $\epsilon(\boldsymbol{q}, \omega)^{-1}$ as a sum of poles. The approach yields both the quasiparticle self-energy to account for extrinsic losses and the many-body amplitude factor $S_{0}^{2}$ to account for intrinsic losses and interference terms. The validity of the self-energy model was checked by comparison with more detailed, first principles calculations. [15] We find that $S_{0}^{2}$ is nearly energy independent over a broad range. Calculations with the many-pole model are shown to improve agreement with experimental results for the near edge XAS of several materials. In addition we find that our model is consistent with the plasmon-pole model when applied to the extended (EXAFS) region, which is an important step toward quantitative full spectrum calculations. A drawback of the present model is that it does not fully account for the contribution due to the particlehole continuum at low energies. Thus the current approach may be expected to give better results for materials with broad loss functions, since in these cases the self-energy will be dominated by plasmon-like excitations even at low energies. Other improvements would be to represent the energy loss function as a sum of broadened poles with momentum transfer dependent broadening, and to better account for the particle-hole continuum; efforts along these lines are in progress. Finally we note that the development of $a b$ initio calculations of inelastic losses here, together with the recently developed ab initio Debye Waller factor calculations [40] yields improved first principles calculations of XAS from structural coordinates alone, without phenomenological models or the need to fit theoretical model self-energy, mean free path, or many-body amplitude parameters.

This work was supported by DOE Grant DE-FG03-97ER45623 (JJR,MPP), NIH NCRR BTP Grant RR-01209 (JJK), NIST Grant 70 NAMB 2H003 (APS), and Academy of Finland Contract No 201291/205967/110571 (JAS) and was facilitated by the DOE Computational Materials Science Network. 


\section{PLASMON-POLE SELF-ENERGY}

Here we give a more complete description of the plasmon-pole (PP) model of Hedin and Lundqvist. [7, 20, 21] We begin with the GW approximation for the self-energy [7]

$$
\Sigma\left(\boldsymbol{r}, \boldsymbol{r}^{\prime}, E\right)=i \int \frac{d \omega}{2 \pi} G\left(\boldsymbol{r}, \boldsymbol{r}^{\prime}, E-\omega\right) W\left(\boldsymbol{r}, \boldsymbol{r}^{\prime}, \omega\right) .
$$

Here $G$ is the single-particle Greens function, which has a spectral representation

$$
G\left(\boldsymbol{r}, \boldsymbol{r}^{\prime}, E\right)=\sum_{i} \frac{\phi_{i}(\boldsymbol{r}) \phi_{i}^{*}\left(\boldsymbol{r}^{\prime}\right)}{E-E_{i}+i \delta \operatorname{sgn}\left(E_{i}-E_{F}\right)},
$$

and $W$ is the dynamically screened coulomb potential,

$$
\begin{aligned}
W\left(\boldsymbol{r}, \boldsymbol{r}^{\prime}, \omega\right) & =\int d^{3} r^{\prime \prime} \epsilon\left(\boldsymbol{r}, \boldsymbol{r}^{\prime \prime}, \omega\right)^{-1} V\left(\boldsymbol{r}^{\prime \prime}, \boldsymbol{r}^{\prime}\right), \\
V\left(\boldsymbol{r}, \boldsymbol{r}^{\prime}\right) & =\frac{1}{\left|\boldsymbol{r}-\boldsymbol{r}^{\prime}\right|}
\end{aligned}
$$

Here $V$ is the bare Coulomb potential and $\epsilon^{-1}$ is the inverse dielectric matrix. Using the Green's function for a homogeneous electron gas, the self-energy in the momentum representation is given by Eq. (11). In frequency space the imaginary part of the inverse dielectric function (i.e., the loss function of the electron gas) is modeled as a single pole at

$\omega(q)=\left[\omega_{p}^{2}+a q^{2}+b q^{4}\right]^{1 / 2}$, where the coefficients of the dispersion $a=k_{F}^{2} / 3$ and $b=1 / 4$ are chosen to give the Thomas-Fermi potential at low frequency, as well as the correct high momentum transfer limit. [7, 21] This gives an inverse dielectric function whose imaginary part is given by

$$
L(\boldsymbol{q}, \omega)=-\operatorname{Im}\left[\epsilon(\boldsymbol{q}, \omega)^{-1}\right]=\pi \omega_{p}^{2} \delta\left[\omega^{2}-\omega(q)^{2}\right] .
$$

The real part of the loss function can be obtained via a Kramers-Kronig transform

$$
\begin{aligned}
\operatorname{Re}\left[\epsilon(\boldsymbol{q}, \omega)^{-1}\right] & =1-\frac{1}{\pi} \int_{0}^{\infty} d \omega^{\prime} \frac{2 \omega^{\prime}}{\omega^{2}-\omega^{\prime 2}} \operatorname{Im}\left[\epsilon\left(\boldsymbol{q}, \omega^{\prime}\right)^{-1}\right] \\
& =1+\frac{\omega_{p}^{2}}{\omega^{2}-\omega(q)^{2}}
\end{aligned}
$$

Inserting these results into Eq. (1) then yields two terms: the first term can be integrated analytically and gives a static Hartree-Fock exchange potential $\Sigma_{H F}$

$$
\Sigma_{H F}(k)=-\frac{k_{F}}{\pi}\left[1+\frac{k_{F}^{2}-k^{2}}{2 k k_{F}} \ln \left|\frac{k_{F}+k}{k_{F}-k}\right|\right] .
$$


The second term, denoted by $\Sigma_{d}\left(\boldsymbol{k}, E ; \omega_{p}\right)$, is the dynamically screened exchange-correlation contribution, which can be interpreted as the one loop diagram containing the electron propagator $G$ and a boson (plasmon) propagator

$$
D(\boldsymbol{q}, \omega)=\frac{2 \omega(q)}{\omega^{2}-\omega(q)^{2}+i \delta} .
$$

Thus the dynamic term $\Sigma_{d}$ arises from the creation of virtual bosons which interact with the photoelectron via an effective coupling $|g(q)|^{2}=\omega_{p}^{2} V(q) / 2 \omega(q)$. The integral over $\omega$ and solid angle in Eq. (1) can be done analytically, so that $\Sigma_{d}(\boldsymbol{k}, E)$ is given by a onedimensional integral over $|\boldsymbol{q}|$. The resulting expression for the self-energy is quite lengthy and is not reproduced here, but can be found in Eq. (13) of Ref. 21.

\section{EQUIVALENCE OF SELF-ENERGY FORMULAE}

In this appendix we demonstrate that self-energy expressions of Hedin and Lundqvist and of Quinn and Farrell are essentially equivalent, except for slight differences the approximations used. We start with the self-energy of an electron gas within the $G W$ approximation as given by Hedin and Lunqvist[20] in Eq. (11). This expression can be split into two terms. The Hartree-Fock exchange potential $\Sigma_{H F}$, and the dynamically screened exchange-correlation potential $\Sigma_{d}$ which includes the dynamic response proportional to $\left[\epsilon(\boldsymbol{q}, \omega)^{-1}-1\right]$.

$$
\Sigma(\boldsymbol{k}, E)=\Sigma_{H F}(\boldsymbol{k})+\Sigma_{d}(\boldsymbol{k}, E)
$$

and

$$
\begin{aligned}
\Sigma_{d}(\boldsymbol{k}, E) & =i \int \frac{d^{3} q}{(2 \pi)^{3}} \frac{d \omega}{2 \pi} V(q)\left[\epsilon(\boldsymbol{q}, \omega)^{-1}-1\right] \\
& \times \frac{1}{E-\omega-E_{\boldsymbol{k}-\boldsymbol{q}}+i\left(|\boldsymbol{k}-\boldsymbol{q}|-k_{F}\right) \delta} .
\end{aligned}
$$

If we rewrite $\epsilon(\boldsymbol{q}, \omega)^{-1}$ in its spectral representation

$$
\epsilon(\boldsymbol{q}, \omega)^{-1}=1-1 / \pi \int d \omega^{\prime} \frac{2 \omega^{\prime}}{\omega^{2}-\left(\omega^{\prime}-i \delta\right)^{2}} \operatorname{Im}\left[\epsilon(\boldsymbol{q}, \omega)^{-1}\right],
$$

Eq. (30) becomes

$$
\begin{aligned}
& \Sigma_{d}(\boldsymbol{k}, E)=-\frac{i}{\pi} \int_{0}^{\infty} \int \frac{d^{3} q}{(2 \pi)^{3}} 2 \omega^{\prime} V(q) \operatorname{Im}\left[\epsilon\left(\boldsymbol{q}, \omega^{\prime}\right)^{-1}-1\right] \\
& \times \int \frac{d \omega}{2 \pi} \frac{1}{\omega^{2}-\left(\omega^{\prime}-i \delta\right)^{2}} \frac{1}{E-\omega-E_{\boldsymbol{k}-\boldsymbol{q}}+i\left(|\boldsymbol{k}-\boldsymbol{q}|-k_{F}\right) \delta} .
\end{aligned}
$$


The integral over $\omega$ can be performed by deforming the contour to the imaginary axis and including residues of the Greens function when necessary. The integral along the imaginary axis is purely real, thus the imaginary part of the self-energy is given by the imaginary part of the contribution from the residues of the poles in the Greens function. The result can then be split into two terms. One arises from the particle contribution and occurs for energies greater than the Fermi energy

$$
\begin{aligned}
\operatorname{Im}[\Sigma(\boldsymbol{k}, E)] & =\int \frac{d^{3} q}{(2 \pi)^{3}} \Theta\left(\operatorname{Im}\left[\Delta E_{\boldsymbol{k}-\boldsymbol{q}}\right]\right) \Theta\left(\operatorname{Re}\left[\Delta E_{\boldsymbol{k}-\boldsymbol{q}}\right]\right) \\
& \times \frac{1}{q^{2}} \operatorname{Im}\left[\epsilon\left(\boldsymbol{q}, \Delta E_{\boldsymbol{k}-\boldsymbol{q}}\right)^{-1}\right],
\end{aligned}
$$

where

$$
\Delta E_{\boldsymbol{k}-\boldsymbol{q}}=E-E_{\boldsymbol{k}-\boldsymbol{q}}+i \delta\left(|\boldsymbol{k}-\boldsymbol{q}|-k_{F} .\right.
$$

The other is associated with the hole contribution where the energy is less than the Fermi energy

$$
\begin{aligned}
\operatorname{Im}[\Sigma(\boldsymbol{k}, E)] & =-\int \frac{d^{3} q}{(2 \pi)^{3}} \Theta\left(-\operatorname{Im}\left[\Delta E_{\boldsymbol{k}-\boldsymbol{q}}\right]\right) \Theta\left(-\operatorname{Re}\left[\Delta E_{\boldsymbol{k}-\boldsymbol{q}}\right]\right) \\
& \times \frac{1}{q^{2}} \operatorname{Im}\left[\epsilon\left(\boldsymbol{q}, \Delta E_{\boldsymbol{k}-\boldsymbol{q}}\right)^{-1}\right] .
\end{aligned}
$$

Quinn and Ferrell make the further approximation that $E=k^{2} / 2$, which yields the formula derived in Ref. 41 and is used as a starting point by Penn. [11, 42] Thus, Penn's formulation is equivalent to that of Hedin and Lundqvist [20, 21] with zeroth order approximations for the quasiparticle energy and renormalization constant.

[1] J. J. Rehr and R. C. Albers, Rev. Mod. Phys. 72, 621 (2000).

[2] J. J. Rehr and A. L. Ankudinov, Coordination Chemistry Reviews 249, 131 (2005).

[3] J. Mustre de Leon, J. J. Rehr, S. I. Zabinsky, and R. C. Albers, Phys. Rev. B 44, 4146 (1991).

[4] J. J. Rehr, J. J. Kas, M. P. Prange, F. D. Vila, A. L. Ankudinov, L. W. Campbell, and A. P. Sorini (2006), arXiv:cond-mat/0601241.

[5] A. Filipponi, A. Di Cicco, and C. R. Natoli, Phys. Rev. B 52, 15122 (1995).

[6] Y. Joly, Phys. Rev. B 63, 125120 (2001).

[7] L. Hedin and S. Lundqvist, Solid State Phys. 23, 1 (1969). 
[8] F. Aryasetiawan and O. Gunnarsson, Rep. Prog. Phys 61, 237 (1998).

[9] J. C. Inkson, Many-body Theory of Solids (Plenum Press, 1984).

[10] P. Horsch, W. von der Linden, and W.-D. Lukas, Solid State Communications 62, 359 (1987).

[11] D. R. Penn, Phys. Rev. B 35, 482 (1987).

[12] D. R. Penn, Phys. Rev. B 13, 5248 (1975).

[13] G. E. Engel and B. Farid, Phys. Rev. B 47, 15931 (1993).

[14] G. E. Engel, B. Farid, C. M. M. Nex, and N. H. March, Phys. Rev. B 44, 13356 (1991).

[15] J. A. Soininen, J. J. Rehr, and E. L. Shirley, J. Phys.: Condens. Matter 15, 2573 (2003).

[16] W. G. Albur, L. Jönsson, and J. W. Wilkins, Solid State Physics 54, 1 (2000).

[17] A. P. Sorini, J. J. Kas, J. J. Rehr, M. P. Prange, and Z. H. Levine, Physical Review B 74, $165111(2006)$.

[18] M. P. Prange, G. Rivas, A. Ankudinov, and J. Rehr, unpublished.

[19] M. P. Prange, G. Rivas, and J. Rehr, Table of Optical Constants for $\mathrm{Mg}, \mathrm{Al}, \mathrm{Cu}, \mathrm{Ag}, \mathrm{Au}, \mathrm{Bi}$ and C. (World Wide Web, http://leonardo.phys.washington.edu/feff/opcons/, 2005).

[20] B. I. Lundqvist, Phys. kondens. Materie. 6, 193 (1967).

[21] B. I. Lundqvist, Phys. kondens. Materie. 6, 207 (1967).

[22] L. Campbell, L. Hedin, J. J. Rehr, and W. Bardyszewski, Physical Review B 65, 064107 (2002).

[23] W. Bardyszewski and L. Hedin, Physica Scripta 32, 439 (1985).

[24] L. Hedin, Physica B 158, 344 (1989).

[25] A. L. Ankudinov, B. Ravel, J. J. Rehr, , and S. D. Conradson, Phys. Rev. B 58, 7565 (1998).

[26] A. L. Ankudinov and J. J. Rehr, Phys. Rev. B 62, 2437 (2000).

[27] Z. H. Levine and S. G. Louie, Phys. Rev. B 25, 6310 (1982).

[28] M. S. Hybertsen and S. G. Louie, Phys. Rev. B 34, 5390 (1986).

[29] M. S. Hybertsen and S. G. Louie, Phys. Rev. B 37, 2733 (1988).

[30] G. Cappellini, R. Del Sole, L. Reining, and F. Bechstedt, Phys. Rev. B 47, 9892 (1993).

[31] W. von der Linden and P. Horsch, Phys. Rev. B 37, 8351 (1988).

[32] A. L. Ankudinov and J. J. Rehr, Colloque C2, Supplément au Journal de Physique III d'avril C2-121 (1997).

[33] J. A. Soininen, J. J. Rehr, and E. L. Shirley, Physica Scripta T115, 243 (2005).

[34] S. Tanuma, T. Shiratori, T. Kimura, K. Goto, S. Ichimura, and C. J. Powell, Surf. Interface 
Anal. 37, 833 (2005).

[35] D. E. Sayers, E. A. Stern, and F. W. Lytle, Phys. Rev. Lett. 27, 1204 (1971).

[36] J. J. Rehr, Radiation Physics and Chemistry 75, 1547 (2006).

[37] M. Newville, private communication.

[38] T. T. Fister, G. T. Seidler, J. J. Rehr, J. J. Kas, W. T. Elam, J. O. Cross, and K. P. Nagle, Phys. Rev. B 75, 174106 (2007).

[39] B. Ravel and M. Newville, Journal of Synchrotron Radiation 12, 537 (2005).

[40] F. D. Vila, J. J. Rehr, H. H. Rossner, and H. J. Krappe, Phys. Rev. B 76, 014301 (2007).

[41] J. J. Quinn and R. A. Ferrell, Phys. Rev. 112, 812 (1958).

[42] J. J. Quinn, Phys. Rev. 126, 1453 (1962). 